УДК 536.244:621.438

\title{
КОМПЬЮТЕРНОЕ МОДЕЛИРОВАНИЕ ДВУХ ПЕРСПЕКТИВНЫХ ОДНОРЯДНЫХ СХЕМ ПЛЕНОЧНОГО ОХЛАЖДЕНИЯ
}

\author{
Халатов А.А. ${ }^{1,2}$, академик НАН Украины, Панченко Н.А. ${ }^{1,2}$, канд. техн. наук, Макаренко В.А. ${ }^{2}$, \\ Спасенко М.И. ${ }^{2}$ \\ ${ }^{1}$ Институт технической теплофизики НАН Украины, ул. Желябова, 2а, Киев, 03056, Украина \\ ${ }^{2}$ НТУУ «Киевский политехнический институт имени Игоря Сикорского», пр. Победы, 37, Киев, 03056, Украина
}

Наведено результати теоретичного дослідження ефективності плівкового охолодження однорядних схем 3 подачею охолоджувача через отвори в траншеї, напівсферичні поглиблення, а також через традиційні круглі похилі отвори.

Библ. 7, рис.5.
Представлены результаты теоретического исследования эффективности пленочного охлаждения однорядных схем с подачей охладителя через отверстия в траншее, полусферические углубления, а также традиционные круглые наклонные отверстия.
The results are given towards the theoretical investigation of the film cooling efficiency of single-row hole systems with the supply of a coolant through holes arranged in the trench, hemispherical dimples, as well as through traditional cylindrical inclined holes.

Ключевые слова: пленочное охлаждение, полусферические углубления, траншея, параметр вдува, эффективность пленочного охлаждения, компьютерное моделирование.

$d$ - диаметр отверстия вдува;

$D$ - диаметр полусферических углублений;

$h$ - высота траншеи;

$L$ - длина отверстия вдува;

$m=w_{2} \rho_{2} / w_{1} \rho_{1}-$ параметр вдува;

$T$ - температура;

$t$ - поперечный шаг расположения отверстий;

$w$ - скорость потока;

\section{Введение}

Пленочное охлаждение является основным способом внешнего охлаждения лопаток первых ступеней высокотемпературных газовых турбин. На сегодня в мире выполнен большой объем экспериментальных и теоретических исследований в этой области, что позволило создать многие типы энергетических установок. Однако практика показала, что традиционная схема пленочного охлаждения с подачей охладителя через наклонные круглые отверстия характеризуется рядом недостатков, основной из которых - возникновение «почечных» вихрей, что приводит к снижению эффективности пленочного охлаждения, особенно при $m>1,0$. Поэтому поиск альтернативных схем пленочного охлаждения с высокой $x, y, z-$ прямоугольные координаты;

$\alpha$ - угол наклона отверстия к поверхности охлаждения; $\eta=\left(T_{1}-T_{a w}\right)\left(T_{1}-T_{2}\right)-$ эффективность пленочного охлаждения.

\section{Нижние индексы:}

$a w$ - параметры на адиабатической стенке;

2 - параметры вдуваемого потока;

1 - параметры основного потока.

теплофизической эффективностью, более низким расходом охладителя и несложной технологией изготовления сегодня представляет одну из актуальных научно-технических проблем газотурбостроения. Такие исследования проводятся в ведущих газотурбинных центрах различных стран. Анализ опубликованных работ [1-4] показал, что наибольший практический интерес для газотурбостроения Украины представляет подача охладителя в полусферические углубления [5] (предложена и запатентована в ИТТФ НАНУ) и траншею. Эти конфигурации рассмотрены в настоящей работе, их схемы представлены на рис. 1 - традиционный ряд наклонных круглых отверстий (рис. $1, a)$, подача охладителя в траншею (рис. 1,6$)$ и полусферические углубления (рис. 1, в).
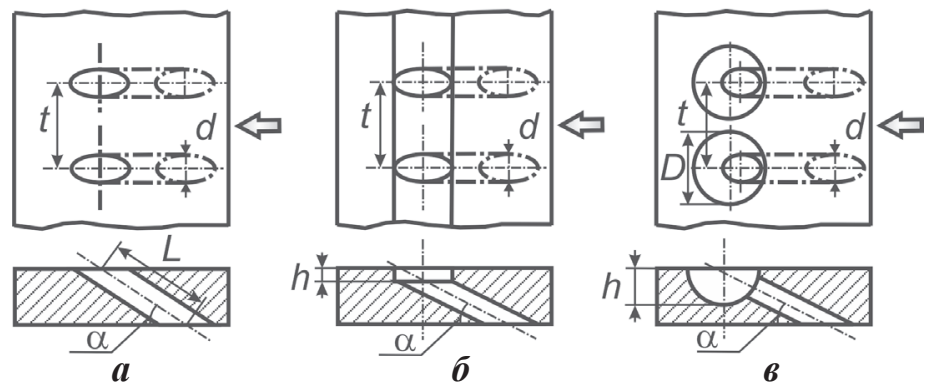

Рис. 1. Исследованные способы плёночного охлажеения: а-традицонный ряд наклонных отверстий; б-отверстия в траншее; в -отверстия в полусферических углублениях. 
Целью настоящеей работы является сравнительное исследование эффективности пленочного охлаждения двух однорядных схем пленочного охлаждения при подаче охладителя через отверстия в траншее и полусферических углублениях, а также через традиционные круглые наклонные отверстия. Исследование выполнено с использованием коммерческого пакета ANSYS CFX 14.

\section{Компьютерная модель}

Исследование выполнено для идентичных геометрических моделей пленочного охлаждения, отличающихся только формой поверхностных углублений, одна из которых для схемы с подачей охладителя в траншею (рис. 1, б) представлена на рис. 2. Она представляет собой канал прямоугольного сечения, в который охладитель подается из внешнего объема (пленума) через отверстия, расположенные в траншее (рис. 2). Схема расположения отверстий и их геометрические размеры были приняты такими же, как и в экспериментальном исследовании [6]. Детальное описание экспериментальной установки приведено в работе [6]. Геометрические параметры были приняты следующими: диаметр подающих цилиндрических отверстий $d=3,2$ мм; поперечный шаг $t=9,6$ мм $(t / d=3,0)$; высота траншеи $h=2,4$ мм $(h / d=0,75)$, диаметр сферических углублений $D=8 \mathrm{мm} ;(h / D=0,5)$. Угол наклона отверстий к поверхности $\alpha=30^{\circ}$.

Граничные условия на входе и выходе были заданы близкими к условиям выполненных экспериментов [6], расположение областей задания граничных условий представлено на рис. 2. Температура основного потока на входе составляла $25^{\circ} \mathrm{C}$, вдуваемого - $72{ }^{\circ} \mathrm{C}$. На входе в канал средняя скорость основного потока задавалась равной 37 м/с. Расчеты выполнены в диапазоне изменения параметра вдува от 0,5 до 2,0. Интенсивность турбулентности основного потока перед участком вдува задавалась равной 1 \%. На выходе из канала среднее статическое давление задавалось стандартным и равным 101300 Па. Расчеты проводились при числе Рейнольдса $\mathrm{Re}_{d}$, определенном по эквивалентному диаметру канала и скорости основного потока, равном $1,25 \times 10^{5}$.

Использовалось неструктурированная комбинированная расчетная сетка, у поверхности пластины, около отверстий и вблизи стенок пленума выполнено локальное сгущение расчетной сетки размерностью в 20 ячеек. Значение параметра $y^{+}$во всех расчетах изменялось в диапазоне от 0,5 до 1,1. Для сравнения использованы данные для традиционной схемы с одним рядом цилиндрических наклонных отверстий. Все расчёты выполнены с использованием комбинированной SST модели турбулентности, представляющей собой суперпозицию $k$ - $\omega$ модели в пристеночной области и $k$ - $\varepsilon$ модели вдали от стенки. Выбор SST модели турбулентности связан с её способностью достаточно точно моделировать газодинамику и теплообмен сложных пристеночных течений, что было показано ранее при выполнении тестовых расчётах с использованием различных моделей турбулентности [7].

\section{Результаты и их анализ}

На рис. 3 представлены результаты расчетов средней эффективности пленочного охлаждения для исследованных схем. Как следует, при малых значениях $m=0,5$ и на основном участке $x / d>20$ все схемы сравнимы по эффективности плёночного охлаждения. Увеличение эффективности плёночного охлаждения при малых значениях параметра вдува наблюдается на начальном и стабилизационном участке $(x / d=0 \ldots 20)$ для схем с подачей охладителя в траншею. При умеренных и высоких значениях параметра вдува $(m \geq 1,0)$ различие в эффективности для рассмотренных схем плёночного охлаждения становится существенным, особенно для схем с подачей охладителя в траншею (рис. 1, б), которая показывает наибольшую эффективность плёночного охлаждения.

На рис. 4 представлены поля эффективности пленочного охлаждения при $m=0,5 ; 1,0 ; 1,5$ и 2,0. За традиционной схемой вдува (рис. $4, a$ ) струя охладителя имеет вид узкой полосы за отверстиями. При вдуве через отверстия в полусферических углублениях (рис. 4, в) благодаря предварительному расширению струи в углублении наблюдается более равномерное распределение охладителя по поверхности. При вдуве через отверстия в траншее (рис. 4, б) охладитель достаточно равномерно растекается по всей ширине поверхности пластины.

На рис. 5 показаны поля температур и проекций векторов скорости в поперечной плоскости на расстоя-

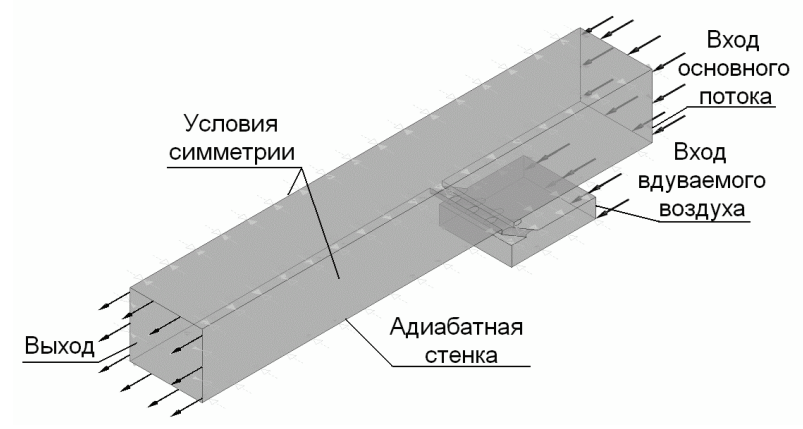

Рис. 2. Геометрическая 3D-модель плёночного охлаждения плоской поверхности при подаче охладителя через отверстия в траншее. 
ниях $x / d=2 ; 5$ и 10 при $m=0,5 ; 1,0 ; 1,5$ и 2,0. За рядами цилиндрических отверстий (рис. 5, a) отчетливо видны характерные вихревые структуры в форме парного («почечного») вихря. При вдуве охладителя в полусферические углубления (рис. 5,6 ) эти вихревые структуры имеют меньшую интенсивность. Аналогичный эффект наблюдается и для схемы с подачей охладителя в тран- шею (рис. 5, в), однако в этом случае снижение интенсивности и масштаба «почечной» вихревой структуры выражено еще более существенное. При таком способе организации плёночного охлаждения защитная плёнка не отрывается от поверхности пластины, и вся поверхность достаточно равномерно покрывается плёнкой охладителя.

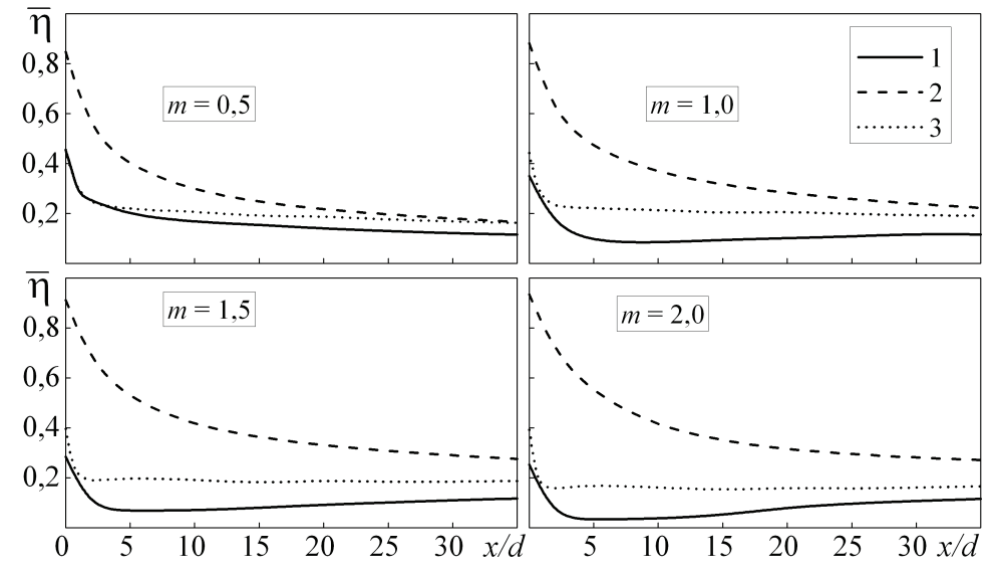

Рис. 3 Средняя по ширине эффективность пленочного охлаждении:

1 - традиционный ряд наклонных отверстий; 2-отверстия в траншее; 3 -отверстия в полусферических углублениях.

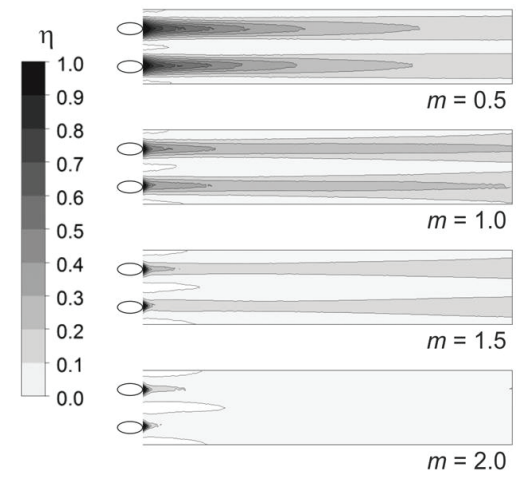

a)
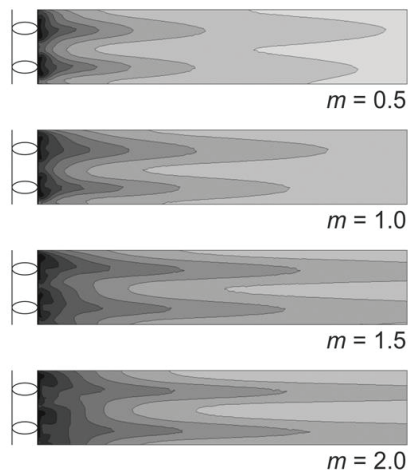

б)
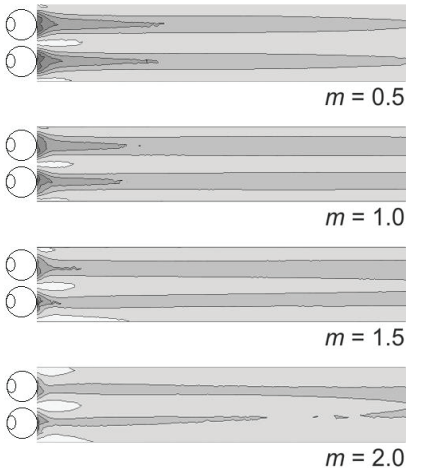

в)

Рис. 4. Изолинии локальной эффективности пленочного охлажждения за традиционными отверстиями (а), отверстиями в траншее (б) и отверстиями в полусферических углублениях (в) на участке $0 \leq x / d \leq 30$.

\section{Выводы}

Выполнено сравнительное теоретическое исследование эффективности пленочного охлаждения и физической структуры потока за двумя перспективными однорядными схемами пленочного охлаждения с подачей охладителя через отверстия в траншее и отверстия в полусферических углублениях.

Основными причинами увеличения эффективности пленочного охлаждения за перспективными схемами пленочного охлаждения является предварительное рас- ширение струи охладителя, снижение интенсивности и масштаба парной вихревой структуры и отсутствие отрыва струи от поверхности.

Из рассмотренных схем плёночного охлаждения наибольшей эффективностью охлаждения обладает схема с подачей охладителя в поперечную траншею, поскольку вся поверхность достаточно равномерно покрывается пленкой охладителя и снижается негативное влияние вторичных вихревых структур. 


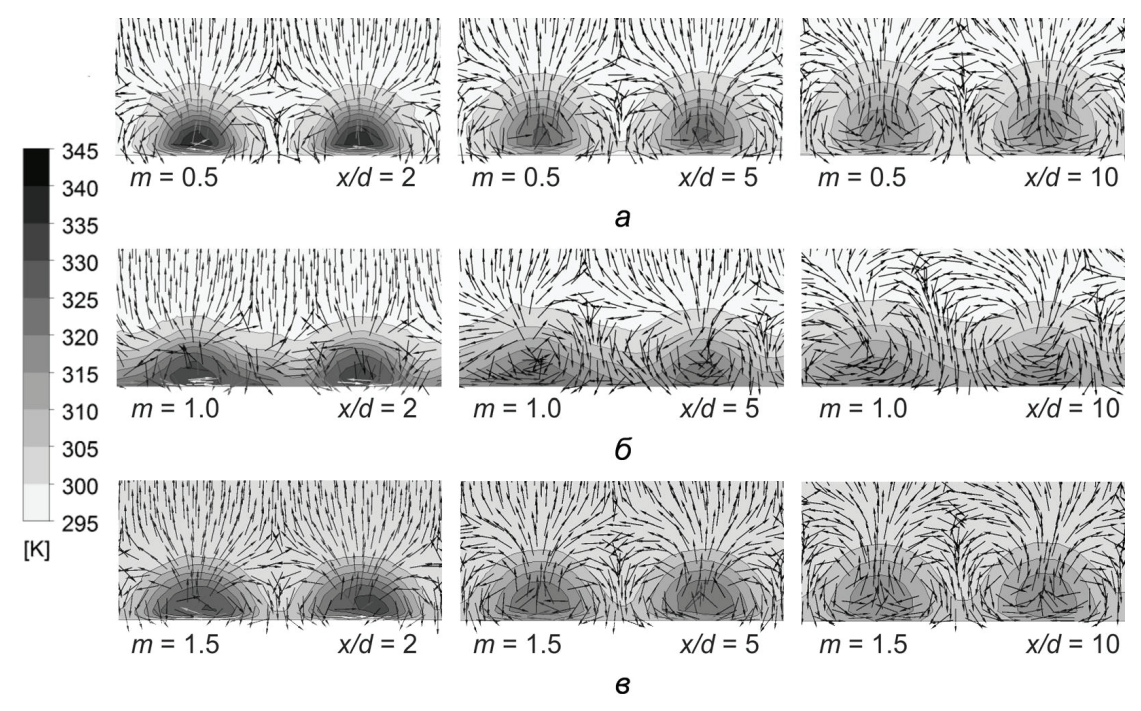

Рис. 5. Температурные поля и проекции векторов скорости на плоскость YZ:

a-традиционые отверстия; б-отверстия в траншее; в -отверстия в полусферических углублениях.

\section{ЛИТЕРАТУРА}

1. Goldstein R.J., Eckert E.R.G. Effects of hole geometry and density on three-dimensional film cooling // Int. J. of Heat and Mass Transfer. - 1974. - V.17, №5. P.595-607.

2. Dittmar J., Schulz A., Wittig S. Assessment of various film cooling configurations including shaped and compound angle based on large scale experiments// Journal of Turbomachinery. - 2003. - V. 125, №1 - - P. 57-64.

3. Lu Y., Dhungel A., Ekkad S.V., Bunker R.S. Effect of trench width and depth on film cooling from cylindrical holes embedded in trenches. // Journal of Turbomachinery. 2009. - V. 131. Paper 011003. 13 p.

4. Khalatov A.A., Borisov I.I., Panchenko N.A., Dashevsky Yu.J., Kovalenko A.S. Flat plate film cooling from a double jet holes: influence of free-stream turbulence and flow acceleration / // Thermophysics and Aeromechanics. 2014. - V. 21. № 5. - P. 545-552.

5. Пат. 47749 України. Спосіб плівкового охолодження та пристрій для його здійснення // Халатов А.А., Варганов І.С., Коваленко Г.В.; заявитель и патентообладатель Институт технической теплофизики. №2001096063; заявл. 03.09.01 ; опубл. 15.12.04, Бюл.№ 12, 2014. -3 с. : ил.

6. Khalatov A.A., Borisov I.I., Dashevskiy Yu.Ya., Kovalenko A.S., Shevtsov S.V. Flat plate film cooling from a single-row inclined holes embedded in a trench: effect of external turbulence and flow acceleration// Thermophysics and Aeromechanics. - 2013. - V. 20, №. 6. - P.713-719.

7. Goldstain R.J., Jin P., Olson R.L. Film cooling effectiveness and mass/heat transfer coefficient downstream of one row of discrete holes// Journal of Turbomachinary. 1999. -V. 121. - P. 225-232. 


\section{COMPUTER SIMULATION OF TWO PERSPECTIVE SINGLE-ROW HOLE SYSTEMS OF FILM COOLING}

Khalatov A.A., ${ }^{1,2}$, Panchenko N.A. ${ }^{1,2}$, Makarenko V.A. ${ }^{2}$, Spasenko M.I. ${ }^{2}$

${ }^{1}$ Institute of Engineering Thermophysics of National Academy of Sciences of Ukraine, Zhelyabova str., 2a, Kyiv, 03056, Ukraine

${ }^{2}$ National Technical University of Ukraine «Igor Sikorsky Kyiv Politechnic Institute», pr. Pobedy, 37, Kyiv, 03056, Ukraine

The comparative theoretical investigation of two prospective film cooling configuration was carried out at the coolant supply through holes arranged in the trench and hemispherical dimples. The numerical simulation was made using ANSYS CFX 14 computer code along with SST turbulence model, the blowing ratio was varied from 0.5 to 2.0. The data for the traditional cooling scheme with one row of inclined cylindrical holes were used for comparison. The results showed that at the identical blowing ratio, the efficiency of the investigated cooling schemes is significantly greater than the efficiency of the traditional scheme having the same relative arrangement pitch of the holes $t / d=3.0$. The investigated schemes reduce the length of the flow separation zone and the intensity of pair ("kidney") vortex. The coolant supply into the trench demonstrates the greatest average film cooling efficiency.

References 7, figures 5 .

Key words: film cooling, hemispherical dimples, trench, blowing ratio, film cooling effectiveness, numerical simulation.
1. Goldstein R.J., Eckert E.R.G. Effects of hole geometry and density on three-dimensional film cooling. Int. J. of Heat and Mass Transfer. 1974. V.17, №5. P.595607.

2. Dittmar J., Schulz A., Wittig S. Assessment of various film cooling configurations including shaped and compound angle based on large scale experiments. Journal of Turbomachinery. 2003. V. 125, №1. P. 57-64.

3. Lu Y., Dhungel A., Ekkad S.V., Bunker R.S. Effect of trench width and depth on film cooling from cylindrical holes embedded in trenches. Journal of Turbomachinery. 2009. V. 131. Paper 011003. 13 p.

4. Khalatov A.A., Borisov I.I., Panchenko N.A., Dashevsky Yu.J. , Kovalenko A.S. Flat plate film cooling from a double jet holes: influence of free-stream turbulence and flow acceleration. Thermophysics and Aeromechanics. 2014. V. 21. № 5. P. 545-552.

5. Khalatov, A. A., Varhanov, I. V. and Kovalenko, H. V. Institute of engineering thermophysics/ Sposib plivkovogo oholodzhennya ta pristriy dlya yogo zdiysnennya [Method of film cooling and appliance for its implementation] State Register of Patents of Ukraine, Kiev, UA, 2004. Pat. No. 47749. (Ukr.)

6. Khalatov A.A., Borisov I.I., Dashevskiy Yu.Ya., Kovalenko A.S., Shevtsov S.V. Flat plate film cooling from a single-row inclined holes embedded in a trench: effect of external turbulence and flow acceleration. Thermophysics and Aeromechanics. 2013. V. 20, №. 6. P.713-719.

7. Goldstain R.J., Jin P., Olson R.L. Film cooling effectiveness and mass/heat transfer coefficient downstream of one row of discrete holes. Journal of Turbomachinary. 1999. V. 121. P. 225-232.

Получено 04.04.2017 Received 04.04.2017 